\title{
Monocytes in Sterile Inflammation: Recruitment and Functional Consequences
}

\author{
Jessica H. Spahn • Daniel Kreisel
}

Received: 8 July 2013 / Accepted: 14 November 2013/Published online: 6 December 2013

(C) L. Hirszfeld Institute of Immunology and Experimental Therapy, Wroclaw, Poland 2013

\begin{abstract}
Monocytes play an important role in initiating innate immune responses. Three subsets of these cells have been defined in mice including classical, nonclassical and intermediate monocytes. Each of these cell types has been extensively studied for their role in infectious diseases. However, their role in sterile injury as occurs during ischemia-reperfusion injury, atherosclerosis, and trauma has only recently been the focus of investigations. Here, we review mechanisms of monocyte recruitment to sites of sterile injury, their modes of action, and their effect on disease outcome in murine models with some references to human studies. Therapeutic strategies to target these cells must be developed with caution since each monocyte subset is capable of mediating either anti- or pro-inflammatory effects depending on the setting.
\end{abstract}

Keywords Monocytes - Sterile inflammation ·

Recruitment - Functional outcome

\section{Introduction}

Monocytes are myeloid-derived cells originating in the bone marrow and maintaining residence in both the peripheral blood and the spleen. These cells, also called mononuclear blood phagocytes, are critical in the

J. H. Spahn · D. Kreisel

Department of Surgery, Washington University in St. Louis,

St. Louis, MO, USA

D. Kreisel ( $\square)$

Department of Pathology and Immunology, Washington

University School of Medicine, 660 South Euclid Avenue,

Campus Box 8234, St. Louis, MO 63110-1013, USA

e-mail: kreiseld@wudosis.wustl.edu inflammatory response to various stimuli, giving rise to tissue-resident macrophages and dendritic cells (DCs). In the mouse, two subtypes of monocytes exist for which there are seemingly corresponding human populations. Classical monocytes in the mouse are characterized by high expression of Ly6C and the chemokine receptor CCR2 as well as intermediate expression of $\mathrm{CD} 43\left(\mathrm{CD} 14^{++} \mathrm{CD} 16^{-}\right.$ in humans), while a nonclassical monocyte population has low expression of both Ly6C and CCR2, but high expression of $\mathrm{CD} 43$ and a separate chemokine receptor, $\mathrm{CX}_{3} \mathrm{CR} 1\left(\mathrm{CD} 14^{+} \mathrm{CD} 16^{++}\right.$in humans). In addition, intermediate monocytes have the phenotype Ly6C high and CD43 high $\left(\mathrm{CD} 14^{++} \mathrm{CD} 16^{+}\right.$in humans) (Geissmann et al. 2003; Ziegler-Heitbrock et al. 2010).

\section{Nonclassical Monocytes}

Geissmann et al. (2003) described these cells as resident monocytes or $\mathrm{Ly}_{6 \mathrm{C}} \mathrm{CCR}^{-} \mathrm{CD} 11 \mathrm{~b}^{\mathrm{hi}} \mathrm{CX} \mathrm{CR}_{3} 1^{\mathrm{hi}}$ cells situated along the endothelium with the role of "patrolling" the vasculature. The same paper termed the other subset inflammatory monocytes. In an effort to avoid the use of functional terms in describing these cells and to create uniformity in the field, a group of monocyte biologists convened and came to the consensus of renaming "inflammatory" cells classical monocytes and "resident" cells nonclassical monocytes (Ziegler-Heitbrock et al. 2010). It has been determined that nonclassical monocytes arise from classical monocytes upon downregulation of Ly6C and are thus the more mature of the two subsets (Sunderkotter et al. 2004). The ligand for $\mathrm{CX}_{3} \mathrm{CR} 1$, $\mathrm{CX}_{3} \mathrm{CL} 1$ or fractalkine, is unique among chemokines in that it is bound to a mucin-like stalk, allowing this chemotactic molecule to be bound to the surface of endothelial 
cells while at the same time extending far enough into the vasculature to make contact with circulating cells (Fong et al. 2000). Unlike classical monocytes, nonclassical monocytes are recruited to tissues even in the absence of inflammation (Geissmann et al. 2003). Nonclassical monocytes have been reported to rapidly extravasate into tissues. One report suggested that these cells are among the first responders to tissue injury and are able to produce both tumor necrosis factor (TNF)- $\alpha$ and interleukin (IL)- $1 \beta$ (Auffray et al. 2007). Nonclassical monocytes are capable of being converted into specialized phagocytic cell types including $\mathrm{CD} 11 \mathrm{c}^{+}$MHC-II ${ }^{+}$dendritic cells in the spleen as well as macrophages in the lung (Geissmann et al. 2003; Landsman and Jung 2007).

\section{Classical Monocytes}

Classical monocytes are characterized by high expression of Ly6C, CCR2, and CD11b with intermediate expression of $\mathrm{CX}_{3} \mathrm{CR} 1$. These monocytes are recruited into inflamed tissue where they are capable of producing both TNF- $\alpha$ and $\mathrm{IL}-1 \beta$. Monocyte emigration from the bone marrow is largely dependent on the chemokine CCL2 (MCP-1) and its receptor CCR2 (Nahrendorf et al. 2007; Serbina and Pamer 2006). Following transendothelial migration, classical monocytes differentiate into either DCs with increased expression of both CD11c and MHC class II or macrophages (Arnold et al. 2007; Geissmann et al. 2003). In vivo experiments have shown that monocyte-derived DCs are capable of inducing $\mathrm{CD} 8^{+} \mathrm{T}$ cell proliferation and regulate activation of $\mathrm{CD}^{+}{ }^{+} \mathrm{T}$ cells (Geissmann et al. 2003; Gelman et al. 2010). Following surgical injury, monocytes are able to produce the neutrophil chemokine, CXCL2, but do not contribute to expression of CXCL1 (Armstrong et al. 2004).

\section{Response to Infectious Agents}

The majority of the literature regarding the role of monocytes responding to infectious pathogens deals with the importance of classical monocytes. However, there is evidence to suggest that $\mathrm{CX}_{3} \mathrm{CR} 1^{\text {hi }}$ nonclassical monocytes are important in defense against Salmonella typhimurium, a pathogenic intestinal bacterium. The role of nonclassical monocytes in response to this pathogen is thought to be in their conversion to tissue-resident DCs expressing high levels of $\mathrm{CX}_{3} \mathrm{CR} 1$. These DCs are able to protrude through the intestinal wall, engulf bacteria, and transport the organism to lymph nodes (Niess et al. 2005). Activation of resident macrophages and DCs in the setting of other mucosal infections such as respiratory syncytial virus and
Legionella pneumophila is also enhanced in the presence of $\mathrm{CX}_{3} \mathrm{CL} 1$ signaling (Johnson et al. 2012; Kikuchi et al. 2005).

Classical monocytes have clearly been shown to be important in response to infection. The elimination of several types of pathogens including bacteria (L. monocytogenes, $M$. tuberculosis), protozoa (T. gondii), and fungi (A. fumigatus) is impaired in mice deficient in CCR2. In response to these infections, classical monocytes can be converted into Tip-DCs (TNF- $\alpha$ and inducible nitric oxide synthase-producing DCs) or directly secrete inflammatory mediators including nitric oxide and IL-12. The role of monocytes in the defense against microbial pathogens has been reviewed extensively (Ingersoll et al. 2011; Serbina et al. 2008).

\section{Sterile Inflammation}

Inflammation is generally thought to occur in response to a pathogenic stimulus. However, many cases of "sterile" inflammation (in the absence of microbial or other infectious material) are known to occur including trauma, atherosclerosis and ischemia-reperfusion injury (as in cases of stroke, myocardial infarction and transplantation) (Chen and Nunez 2010).

Immune responses to infectious pathogens are initiated and amplified by the detection of pathogen-associated molecular patterns, molecules commonly found on the surface of bacteria or released by viruses. Innate immune cells express pattern-recognition receptors, including Tolllike receptors (TLRs), NOD-like receptors, and RIG-I like receptors that bind these molecules and activate innate immune cells to defend against the intruding pathogen (Medzhitov 2001). These same receptors have also been shown to be important in initiating immune responses to injury occurring in a sterile environment, in which case the stimuli are termed damage-associated molecular patterns (DAMPs). Molecules normally sequestered within healthy cells but released upon cell death act as stimuli and include DNA, RNA, high-mobility group box 1 , heat shock proteins (HSP), and cholesterol crystals (Chen and Nunez 2010; Tsan and Gao 2004). As in infectious inflammation, activation of immune cells by DAMPs leads to increased expression of inflammatory cytokines and the recruitment of neutrophils and monocytes.

While the role of monocytes in infectious inflammation has been the topic of many reviews (Serbina et al. 2008; Shi and Pamer 2011) the role of monocytes in sterile inflammation is only recently being appreciated. Although analogies exist between the recruitment of monocytes in sterile and infectious inflammation, there are additional functions of monocytes in repair and healing that are more 
documented in noninfectious inflammation. Here, we will review the role of monocytes in various examples of sterile inflammation in murine systems including the method of their recruitment, mechanisms of action and consequences of their activation.

\section{Mechanisms of Monocyte Recruitment During Sterile Inflammation}

CCL2 (monocyte chemoattractant protein-1) has been shown to be important in recruiting classical monocytes out of the bone marrow and into the bloodstream (Fig. 1). In a paper investigating monocytes in the ischemic liver, monocyte recruitment into the organs of mice lacking CCR2 was virtually eliminated correlating with a concomitant increase of monocytes in the bone marrow (Bamboat et al. 2010). Similar findings have been seen in models of atherosclerosis, in which CCR2 knockout (KO) mice have increased numbers of monocytes in the bone marrow compartment, but decreased numbers at the site of inflammation (Tsou et al. 2007). This is an indication that monocytes recruited to sites of sterile inflammation originate in the bone marrow. A recent paper investigating the effect of the chemotherapeutic drug, cyclophosphamide, demonstrated that classical monocyte egress from the bone marrow is affected in an opposite manner by the $\mathrm{CX}_{3} \mathrm{CL} 1-$ $\mathrm{CX}_{3} \mathrm{CR} 1$ axis (Jacquelin et al. 2013). While CCL2 drives inflammatory monocyte emigration from this compartment, $\mathrm{CX}_{3} \mathrm{CL} 1$ causes these cells to be retained in the bone marrow. In this study, nonclassical monocyte egress from the bone marrow was not affected by the absence of either of these chemokine receptors.

Some studies suggest that recruitment of classical monocytes from the bloodstream to sites of sterile inflammation is largely dependent on the expression of CCL2. This chemokine has been shown to not only cause monocyte chemotaxis but also firm adhesion onto endothelial monolayers (Gerszten et al. 1999; Maus et al. 2002). In a rat model of traumatic brain injury, monocytes migrated across the blood-cerebrospinal fluid barrier in response to CCL2 produced by the choroid plexus epithelial cells (Szmydynger-Chodobska et al. 2012). In another sterile injury model using cerebral ischemia, CCR2-deficient mice have decreased recruitment of classical monocytes (Dimitrijevic et al. 2007). In vitro studies showed that CCL2 was expressed by both brain microvascular endothelial cells and astrocytes (Dimitrijevic et al.

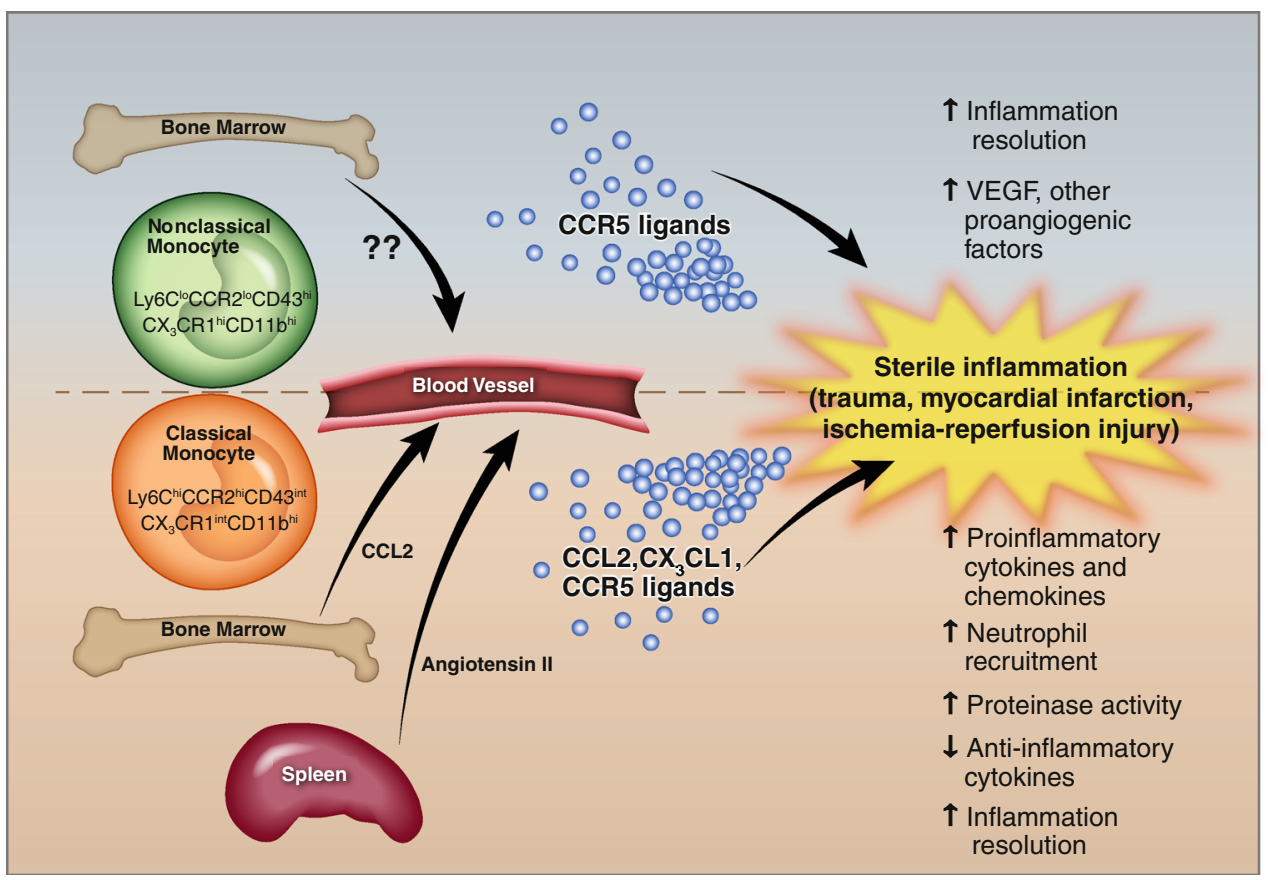

Fig. 1 Two distinct subtypes of murine monocytes exist, nonclassical $\left(\mathrm{Ly} 6 \mathrm{C}^{\mathrm{lo}} \mathrm{CCR} 22^{\mathrm{lo}} \mathrm{CD} 43^{\mathrm{hi}} \mathrm{CX}{ }_{3} \mathrm{CR} 1{ }^{\mathrm{hi}} \mathrm{CD} 11 \mathrm{~b}{ }^{\mathrm{hi}}\right.$ ) and classical (Ly6C ${ }^{\text {hi }} \mathrm{CCR} 2{ }^{\text {hi }} \mathrm{CD} 43^{\text {int }} \mathrm{CX}_{3} \mathrm{CR}_{1}^{\text {int }} \mathrm{CD} 11 \mathrm{~b}^{\text {hi }}$ ). The nonclassical subset originates in the bone marrow and is recruited to sites of inflammation by CCR5 ligands. Classical monocytes reside in the bone marrow, but also have a reservoir in the spleen. Egress from the bone marrow is dependent on CCR2 ligands, while migration out of the spleen is dependent on Angiotensin II. Upon entering the bloodstream, they are recruited to sites of inflammation through CCL2, $\mathrm{CX}_{3} \mathrm{CL} 1$, and CCR5 ligands. While nonclassical monocytes play an important role in the resolution of inflammation and secreting vascular endothelial growth factor $(V E G F)$ and other pro-angiogenic factors, classical monocytes increase production of pro-inflammatory cytokines and chemokines, cause neutrophil recruitment, lead to an increase in proteinase activity, and can also resolve inflammation 
2006). Surprisingly, endothelial cells in this setting also expressed the receptor CCR2, and ligation with CCL2 caused tight junctions in endothelial cells to be disrupted increasing vascular permeability. The CCR2-CCL2 axis in this system has developed to not only directly recruit monocytes to the injured tissue, but also to further facilitate entry of these cells by causing disruption of the endothelial barrier. However, lung transplant studies have shown that while the emigration of monocytes from the bone marrow to the peripheral blood is dependent on CCR2, their migration into graft tissue is independent of this chemokine, which mirrors observations in a model of infection with Listeria monocytogenes (Gelman et al. 2010; Serbina and Pamer 2006).

In addition to the bone marrow, the spleen also provides an important monocyte reservoir. By two separate techniques, Swirski et al. (2009) showed that splenic monocytes rather than bone marrow-derived monocytes populated the ischemic myocardium. Consistent with the notion that CCR2 is necessary for monocyte release from the bone marrow but not from the spleen, monocytes were efficiently released into the bloodstream in CCR2KO mice. In addition, splenectomizing mice prior to ischemia led to an inability of monocytes to populate the inflamed heart tissue (Swirski et al. 2009). Moreover, this study showed the importance of angiotensin II in splenic monocyte egress. A separate study also using myocardial infarction showed that monocytopoiesis in the spleen was dependent on the inflammatory cytokine IL-1 $\beta$ (Leuschner et al. 2012).

Several studies have examined the role of $\mathrm{CX}_{3} \mathrm{CR} 1$ in the recruitment of monocytes during sterile inflammation. Tacke et al. (2007) showed some surprising mechanisms of both classical and nonclassical monocyte recruitment in a mouse model of atherosclerosis. ApoE ${ }^{-/-}$mice fed a high fat diet are susceptible to atherosclerotic lesions and have an increased abundance of monocytes in the blood. This group designed methods for labeling either classical or nonclassical monocytes using fluorescent latex beads. Specific labeling of nonclassical monocytes was achieved by observing bead positive monocytes $8 \mathrm{~h}$ after injection of fluorescent beads. While both subsets of monocytes initially took up these beads, at $8 \mathrm{~h}$, labeling was restricted to only the nonclassical monocytes. Alternatively, specific labeling of classical monocytes was achieved by first depleting all monocytes with clodronate liposomes followed $24 \mathrm{~h}$ later by injection of fluorescent beads. Upon monocyte return, latex bead labeling was restricted to Ly6 $\mathrm{C}^{\text {hi }}$ classical monocytes. Using these methods of monocyte labeling, Tacke et al. (2007) found that both $\mathrm{CCR} 2$ and $\mathrm{CX}_{3} \mathrm{CR} 1$ were required for migration of classical monocytes into atherosclerotic lesions. However, neither of these two chemokine receptors was required for nonclassical monocyte migration, which was dependent on CCR5 (Tacke et al. 2007).

In a model of renal ischemia reperfusion injury, Oh et al. (2008) showed that the chemokine $\mathrm{CX}_{3} \mathrm{CL} 1$ was expressed by kidney endothelial cells within $8 \mathrm{~h}$ of reperfusion. This group also provided in vitro evidence that the increased expression of $\mathrm{CX}_{3} \mathrm{CL} 1$ was due to exposure of endothelial cells to hypoxic conditions. Blocking the interaction of this chemokine with its receptor using a $\mathrm{CX}_{3} \mathrm{CR} 1$ antibody decreased the number of $\mathrm{CD} 11 \mathrm{~b}^{+}$cells (which the authors determined to be macrophages) infiltrating the kidney. In a separate model using cardiac allograft rejection, expression of $\mathrm{CX}_{3} \mathrm{CL} 1$ continued to increase on graft endothelium up to 7 days post-transplant (Robinson et al. 2000). In vitro studies performed by this group demonstrated that the increased chemokine expression on endothelial cells was due to exposure to TNF- $\alpha$. Furthermore, $\mathrm{CX}_{3} \mathrm{CL} 1$ mediated adhesion of peripheral blood mononuclear cells to endothelial cells and treatment of murine heart transplant recipients with neutralizing anti-CX $\mathrm{CX}_{3} \mathrm{CR} 1$ antibodies resulted in prolonged allograft survival.

Other methods of monocyte recruitment to sites of sterile injury have been investigated including a role for matrix metalloproteinase-9 (MMP-9) and CXCL12. Zhang and colleagues (2011) showed that CXCL12 contributes to the recruitment of monocytes to sites of spinal cord injury. Upon entering the tissue, monocytes produced MMP-9, potentially causing the breakdown of the endothelial basement membrane and therefore allowing transmigration of the cells. In these experiments, drugs targeting MMP-9 and CXCL12 as well as the absence of these molecules in genetically deficient mice resulted in decreased recruitment of monocytes to the site of injury (Zhang et al. 2011). Local production of TNF- $\alpha$ at the site of injury may have triggered the expression of MMP-9. Thus, a complex system of chemokines, cytokines and proteolytic enzymes was proposed to be responsible for monocyte migration into the inflamed tissue.

\section{Mechanisms of Monocyte Action During Sterile Inflammation}

As monocytes quickly differentiate into macrophages or DCs upon leaving the bloodstream, it is difficult to isolate monocyte function from that of their tissue-resident counterparts. That being said, the literature does contain various references of direct functions of monocytes in sterile inflammation.

In models of sterile injury in mice deficient in CCR2, where recruitment of monocytes is impaired, levels of proinflammatory mediators at the site of damage are significantly altered. Compared to wild-type mice, CCR2 KO 
mice undergoing cerebral ischemia reperfusion injury had decreased expression of the neutrophil chemoattractant CXCL5 as well as the pro-inflammatory cytokines IL-1 $\beta$ and TNF- $\alpha$ at the site of inflammation. In contrast, expression of cytokines such as IL-4, IL-5 and IL-13 was increased in CCR2 KO animals (Dimitrijevic et al. 2007). While experiments performed in this study do not definitively point to monocytes as the producers of these inflammatory modulators, it does demonstrate that monocytes have a profound effect on the local inflammatory state.

Evidence of direct monocyte expression of pro-inflammatory cytokines has been shown in ex vivo-stimulated monocytes derived from humans following myocardial infarction. These patients have increased serum levels of HSP70, which as mentioned above can act as a DAMP. This endogenous activator of the innate immune system is released upon cardiac damage and can bind to TLR4. Monocytes isolated from patients post-myocardial infarction and incubated with different concentrations of HSP70 show increased expression of inflammatory cytokines including IL-6 and TNF- $\alpha$. Blocking TLR4 in these experiments inhibited monocyte production of these cytokines (Satoh et al. 2006). Mouse models have also demonstrated the importance of monocytes in directly contributing to the inflammatory milieu. Classical monocytes infiltrating livers following ischemia reperfusion injury have increased expression of reactive oxygen species, TNF- $\alpha$ and IL-6 (Bamboat et al. 2010). Depletion of CD11 ${ }^{+}$DCs resulted in increased expression of these proinflammatory mediators specifically in Ly6 $\mathrm{C}^{\text {hi }}$ monocytes suggesting that DCs control detrimental effects of classical monocytes during liver ischemia. In this model, monocytes were largely responsible for ischemia reperfusion injurymediated liver damage (Bamboat et al. 2010).

Regulation of such inflammatory cytokines may in part be through the expression of two interacting proteins, the arrestin protein, $A R R B 2$, and $I \kappa B$ in monocytes. In a renal transplant model, monocytes accumulate in large numbers in the vasculature of rejecting allografts compared to isografts (Grau et al. 2001). In these monocytes, decreased expression of ARRB2 and I $\mathrm{B}$ as measured by immunoblotting was associated with increased $\mathrm{NF} \kappa \mathrm{B}$ signaling and downstream expression of IL-1 $\beta$ and TNF- $\alpha$ (Zakrzewicz et al. 2011).

Monocytes may further contribute to inflammation through the recruitment of neutrophils, which are known to be important mediators of tissue injury during inflammation. Early in vitro studies showed that hypoxic conditions associated with ischemia reperfusion injury caused monocytes to express neutrophil chemoattractants (Metinko et al. 1992). Recent intravital two-photon microscopy studies performed in our laboratory uncovered a role for monocytes in the recruitment of neutrophils into reperfused lung grafts (Kreisel et al. 2010). Injection of clodronate liposomes, which deplete blood-borne phagocytic cells including monocytes, resulted in the inability of neutrophils to traffic into the ischemic lung tissue despite being capable of adhering to the endothelium. This indicates that monocytes regulate the transendothelial migration of neutrophils into sites of sterile inflammation. Furthermore, neutrophils frequently co-localized with monocytes in the transplanted lung tissue raising the possibility that monocytes provide chemotactic cues to neutrophils following their extravasation.

However, monocyte function is not always pro-inflammatory. Activated intravascular monocytes within transplanted renal grafts during rejection may also play a role in dampening inflammation through expression of nonneuronal acetylcholine, which can attenuate ATPmediated signaling in autocrine or paracrine fashion (Hecker et al. 2009). During spinal cord injury, monocytes can have a protective role regulating the resolution of scar tissue. These cells are converted into "resolving/regulatory" macrophages expressing both IL-10 and MMP-13 (Shechter et al. 2009, 2011). Expression of IL-10 by monocytes led to an overall anti-inflammatory state that limited lesion size and prevented resident microglial cell activation (Shechter et al. 2009). Monocytes in spinal cord injury also regulate scar resolution by secreting MMP-13 in response to chondroitin sulfate proteoglycan, a component of the glial scar matrix (Shechter et al. 2011).

Nahrendorf et al. (2007) investigated the role of both classical and nonclassical monocytes in response to myocardial infarction. After cardiac injury, these two subsets were recruited to the tissue in two waves: an initial phase, during which classical monocytes were primarily recruited and a secondary phase during which nonclassical monocytes predominated. During the first phase, Ly6C ${ }^{\text {hi }} \mathrm{CCR} 2^{\text {hi }}$ monocytes exhibited high proteinase activity (presumably to break down the extracellular matrix) as well as proinflammatory TNF- $\alpha$. Ly $6 \mathrm{C}^{\mathrm{lo}} \mathrm{CX}_{3} \mathrm{CR} 1^{\mathrm{hi}}$ monocytes, however, were more important in healing, expressing vascular endothelial growth factor and other pro-angiogenic factors (Nahrendorf et al. 2007).

\section{Monocyte Effect on Outcome of Disease During Sterile Inflammation}

For the majority of examples of sterile inflammation, monocytes have been shown to be detrimental to the outcome of disease. In cases of cerebral ischemia (Dimitrijevic et al. 2007), bronchiolitis obliterans syndrome associated with tracheal transplantation (Belperio et al. 2001) and atherosclerosis (Boring et al. 1998), 
absence or disruption of the CCR2-CCL2 axis was associated with improved outcomes. A recent patient study investigating pancreatic cancer demonstrated that higher monocyte abundance in the blood was correlated with decreased survival over a 5-year period. These monocytes infiltrated the tumors where they presumably became tumor-associated macrophages (TAM) capable of suppressing infiltrating $\mathrm{CD}^{+} \mathrm{T}$ cells. In the same study, a mouse model of pancreatic cancer showed that treatment with a CCR2 inhibitor promoted antitumor immunity associated with a decrease in TAMs and concomitant increase in tumor-infiltrating effector T cells (Sanford et al. 2013).

However, evidence also exists for a role of classical monocytes in the improvement of outcome. Several studies examining skeletal muscle injury illustrated an important role for CCR $2^{\text {hi }}$ monocytes in the repair of tissue. Models using freeze-induced injury and injection of cardiotoxin have demonstrated that recruitment of monocytes (presumably the classical subset due to the use of CCR2 KO mice) improves the functional strength of recovering muscles (Warren et al. 2004, 2005). In the case of cardiotoxin-induced injury, CCR2 KO mice had delayed cellular infiltrates, impaired muscle regeneration, and increased intermuscular adipocytes (Martinez et al. 2010). Furthermore, monocytes (as well as other bone marrow cells and muscle tissue) expressed CCL2 leading to increased expression of insulin-like growth factor, which was important in the healing process (Lu et al. 2011). As discussed above, separate studies on spinal cord injury have shown that infiltration of monocytes to the site of damage is necessary for both controlling inflammation and remodeling of scar tissue (Shechter et al. 2009, 2011).

The literature regarding the effects of nonclassical monocytes on outcomes of diseases is more ambiguous. Studies examining cerebral (Soriano et al. 2002) and renal (Furuichi et al. 2006) ischemia reperfusion injury showed that the absence of $\mathrm{CX}_{3} \mathrm{CR} 1$ signaling correlated with decreased inflammation. In the study of renal ischemia reperfusion injury, absence of this signaling pathway was also associated with less fibrosis. However, other studies indicate that the $\mathrm{CX}_{3} \mathrm{CL} 1-\mathrm{CX}_{3} \mathrm{CR} 1$ axis is associated with improved outcomes. Louvet et al. (2004) demonstrated that increased expression of $\mathrm{CX}_{3} \mathrm{CL} 1$ and its receptor are linked with cardiac allograft tolerance. As mentioned above, $\mathrm{CX}_{3} \mathrm{CR} 1^{\mathrm{hi}}$ monocytes were important in the healing myocardium after infarction (Nahrendorf et al. 2007). Finally, one other study, while not implicating monocytes in the effect, showed that $\mathrm{CX}_{3} \mathrm{CL} 1$ expression was important in angiogenesis and recovery after hindlimb ischemia (Ryu et al. 2008). These reports illustrate the importance of using prudence when considering blockade of these pathways for clinical interventions.

\section{Conclusion}

Presently, there are few drugs to antagonize either CCR2 or $\mathrm{CX}_{3} \mathrm{CR} 1$. Two drugs, RO5234444 and RAP-103, also block the CCR5 receptor and are in pre-clinical trials. Two studies have shown efficacy for these orally active small molecule antagonists. RO5234444 showed improved outcomes in diabetic nephropathy (Sayyed et al. 2011) while RAP-103 attenuated rodent neuropathic pain (Padi et al. 2012). A separate drug called PF-04136309 is a CCR2 kinase antagonist from Pfizer (Xue et al. 2010). Sanford et al. (2013) are beginning Phase $\mathrm{Ib} / \mathrm{II}$ clinical trials with this drug in pancreatic cancer to block classical monocyte infiltration into tumors.

The role of monocytes in sterile inflammation is only starting to be fully appreciated. These cells are obviously very important in exacerbating inflammation in several models of sterile inflammation. However, they may also be critical in the later phases of healing. In addition, the role of monocytes may differ based on different anatomic locations and the nature of the inflammatory stimuli. With this in mind, it will be important to consider the possibility of monocyte inhibition causing both favorable and unfavorable results in different clinical settings.

Acknowledgments D. Kreisel receives support from NIH Grants R01 HL113931 and R01 HL094601.

\section{References}

Armstrong DA, Major JA, Chudyk A et al (2004) Neutrophil chemoattractant genes KC and MIP-2 are expressed in different cell populations at sites of surgical injury. J Leukoc Biol 75:641-648

Arnold L, Henry A, Poron F et al (2007) Inflammatory monocytes recruited after skeletal muscle injury switch into antiinflammatory macrophages to support myogenesis. J Exp Med 204:1057-1069

Auffray C, Fogg D, Garfa M et al (2007) Monitoring of blood vessels and tissues by a population of monocytes with patrolling behavior. Science 317:666-670

Bamboat ZM, Ocuin LM, Balachandran VP et al (2010) Conventional DCs reduce liver ischemia/reperfusion injury in mice via IL-10 secretion. J Clin Invest 120:559-569

Belperio JA, Keane MP, Burdick MD et al (2001) Critical role for the chemokine MCP-1/CCR2 in the pathogenesis of bronchiolitis obliterans syndrome. J Clin Invest 108:547-556

Boring L, Gosling J, Cleary M et al (1998) Decreased lesion formation in CCR2-/- mice reveals a role for chemokines in the initiation of atherosclerosis. Nature 394:894-897

Chen GY, Nunez G (2010) Sterile inflammation: sensing and reacting to damage. Nat Rev Immunol 10:826-837

Dimitrijevic OB, Stamatovic SM, Keep RF et al (2006) Effects of the chemokine CCL2 on blood-brain barrier permeability during ischemia-reperfusion injury. $\mathrm{J}$ Cereb Blood Flow Metab 26:797-810

Dimitrijevic OB, Stamatovic SM, Keep RF et al (2007) Absence of the chemokine receptor CCR2 protects against cerebral ische$\mathrm{mia} /$ reperfusion injury in mice. Stroke 38:1345-1353 
Fong AM, Erickson HP, Zachariah JP et al (2000) Ultrastructure and function of the fractalkine mucin domain in $\mathrm{CX}(3) \mathrm{C}$ chemokine domain presentation. J Biol Chem 275:3781-3786

Furuichi K, Gao JL, Murphy PM (2006) Chemokine receptor CX3CR1 regulates renal interstitial fibrosis after ischemiareperfusion injury. Am J Pathol 169:372-387

Geissmann F, Jung S, Littman DR (2003) Blood monocytes consist of two principal subsets with distinct migratory properties. Immunity $19: 71-82$

Gelman AE, Okazaki M, Sugimoto S et al (2010) CCR2 regulates monocyte recruitment as well as CD4 T1 allorecognition after lung transplantation. Am J Transplant 10:1189-1199

Gerszten RE, Garcia-Zepeda EA, Lim YC et al (1999) MCP-1 and IL8 trigger firm adhesion of monocytes to vascular endothelium under flow conditions. Nature 398:718-723

Grau V, Stehling O, Garn H et al (2001) Accumulating monocytes in the vasculature of rat renal allografts: phenotype, cytokine, inducible no synthase, and tissue factor mRNA expression. Transplantation 71:37-46

Hecker A, Mikulski Z, Lips KS et al (2009) Pivotal advance: upregulation of acetylcholine synthesis and paracrine cholinergic signaling in intravascular transplant leukocytes during rejection of rat renal allografts. J Leukoc Biol 86:13-22

Ingersoll MA, Platt AM, Potteaux S et al (2011) Monocyte trafficking in acute and chronic inflammation. Trends Immunol 32:470-477

Jacquelin S, Licata F, Dorgham K et al (2013) CX3CR1 reduces Ly6Chigh-monocyte motility within, and release from the bone marrow after chemotherapy in mice. Blood 122:674-683

Johnson CH, Miao C, Blanchard EG et al (2012) Effect of chemokine receptor CX3CR1 deficiency in a murine model of respiratory syncytial virus infection. Comp Med 62:14-20

Kikuchi T, Andarini S, Xin H et al (2005) Involvement of fractalkine/ CX3CL1 expression by dendritic cells in the enhancement of host immunity against Legionella pneumophila. Infect Immun 73:5350-5357

Kreisel D, Nava RG, Li W et al (2010) In vivo two-photon imaging reveals monocyte-dependent neutrophil extravasation during pulmonary inflammation. Proc Natl Acad Sci USA 107:18073-18078

Landsman L, Jung S (2007) Lung macrophages serve as obligatory intermediate between blood monocytes and alveolar macrophages. J Immunol 179:3488-3494

Leuschner F, Rauch PJ, Ueno T et al (2012) Rapid monocyte kinetics in acute myocardial infarction are sustained by extramedullary monocytopoiesis. J Exp Med 209:123-137

Louvet C, Heslan JM, Merieau E et al (2004) Induction of fractalkine and $\mathrm{CX} 3 \mathrm{CR} 1$ mediated by host $\mathrm{CD}^{+} \mathrm{T}$ cells in allograft tolerance induced by donor specific blood transfusion. Transplantation 78:1259-1266

Lu H, Huang D, Ransohoff RM et al (2011) Acute skeletal muscle injury: CCL2 expression by both monocytes and injured muscle is required for repair. FASEB J 25:3344-3355

Martinez CO, McHale MJ, Wells JT et al (2010) Regulation of skeletal muscle regeneration by CCR2-activating chemokines is directly related to macrophage recruitment. Am J Physiol Regul Integr Comp Physiol 299:R832-R842

Maus U, Henning S, Wenschuh H et al (2002) Role of endothelial MCP-1 in monocyte adhesion to inflamed human endothelium under physiological flow. Am J Physiol Heart Circ Physiol 283:H2584-H2591

Medzhitov R (2001) Toll-like receptors and innate immunity. Nat Rev Immunol 1:135-145

Metinko AP, Kunkel SL, Standiford TJ et al (1992) Anoxiahyperoxia induces monocyte-derived interleukin-8. J Clin Invest 90:791-798
Nahrendorf M, Swirski FK, Aikawa E et al (2007) The healing myocardium sequentially mobilizes two monocyte subsets with divergent and complementary functions. J Exp Med 204:3037-3047

Niess JH, Brand S, Gu X et al (2005) CX3CR1-mediated dendritic cell access to the intestinal lumen and bacterial clearance. Science 307:254-258

Oh DJ, Dursun B, He Z et al (2008) Fractalkine receptor (CX3CR1) inhibition is protective against ischemic acute renal failure in mice. Am J Physiol Renal Physiol 294:F264-F271

Padi SS, Shi XQ, Zhao YQ et al (2012) Attenuation of rodent neuropathic pain by an orally active peptide, RAP-103, which potently blocks CCR2- and CCR5-mediated monocyte chemotaxis and inflammation. Pain 153:95-106

Robinson LA, Nataraj C, Thomas DW et al (2000) A role for fractalkine and its receptor (CX3CR1) in cardiac allograft rejection. J Immunol 165:6067-6072

Ryu J, Lee CW, Hong KH et al (2008) Activation of fractalkine/ CX3CR1 by vascular endothelial cells induces angiogenesis through VEGF-A/KDR and reverses hindlimb ischaemia. Cardiovasc Res 78:333-340

Sanford DE, Belt BA, Panni RZ et al (2013) Inflammatory monocyte mobilization decreases patient survival in pancreatic cancer: a role for targeting the CCL2/CCR2 axis. Clin Cancer Res 19:3404-3415

Satoh M, Shimoda Y, Akatsu T et al (2006) Elevated circulating levels of heat shock protein 70 are related to systemic inflammatory reaction through monocyte Toll signal in patients with heart failure after acute myocardial infarction. Eur J Heart Fail 8:810-815

Sayyed SG, Ryu M, Kulkarni OP et al (2011) An orally active chemokine receptor CCR2 antagonist prevents glomerulosclerosis and renal failure in type 2 diabetes. Kidney Int 80:68-78

Serbina NV, Pamer EG (2006) Monocyte emigration from bone marrow during bacterial infection requires signals mediated by chemokine receptor CCR2. Nat Immunol 7:311-317

Serbina NV, Jia T, Hohl TM et al (2008) Monocyte-mediated defense against microbial pathogens. Ann Rev Immunol 26:421-452

Shechter R, London A, Varol C et al (2009) Infiltrating blood-derived macrophages are vital cells playing an anti-inflammatory role in recovery from spinal cord injury in mice. PLoS Med 6:e1000113

Shechter R, Raposo C, London A et al (2011) The glial scarmonocyte interplay: a pivotal resolution phase in spinal cord repair. PLoS ONE 6:e27969

Shi C, Pamer EG (2011) Monocyte recruitment during infection and inflammation. Nat Rev Immunol 11:762-774

Soriano SG, Amaravadi LS, Wang YF et al (2002) Mice deficient in fractalkine are less susceptible to cerebral ischemia-reperfusion injury. J Neuroimmunol 125:59-65

Sunderkotter C, Nikolic T, Dillon MJ et al (2004) Subpopulations of mouse blood monocytes differ in maturation stage and inflammatory response. J Immunol 172:4410-4417

Swirski FK, Nahrendorf M, Etzrodt M et al (2009) Identification of splenic reservoir monocytes and their deployment to inflammatory sites. Science 325:612-616

Szmydynger-Chodobska J, Strazielle N, Gandy JR et al (2012) Posttraumatic invasion of monocytes across the blood-cerebrospinal fluid barrier. J Cereb Blood Flow Metab 32:93-104

Tacke F, Alvarez D, Kaplan TJ et al (2007) Monocyte subsets differentially employ CCR2, CCR5, and CX3CR1 to accumulate within atherosclerotic plaques. J Clin Invest 117:185-194

Tsan MF, Gao B (2004) Endogenous ligands of Toll-like receptors. J Leukoc Biol 76:514-519

Tsou CL, Peters W, Si Y et al (2007) Critical roles for CCR2 and $\mathrm{MCP}-3$ in monocyte mobilization from bone marrow and recruitment to inflammatory sites. J Clin Invest 117:902-909 
Warren GL, O'Farrell L, Summan M et al (2004) Role of CC chemokines in skeletal muscle functional restoration after injury. Am J Physiol Cell Physiol 286:C1031-C1036

Warren GL, Hulderman T, Mishra D et al (2005) Chemokine receptor CCR2 involvement in skeletal muscle regeneration. FASEB J 19:413-415

Xue CB, Wang A, Meloni D et al (2010) Discovery of INCB3344, a potent, selective and orally bioavailable antagonist of human and murine CCR2. Bioorg Med Chem Lett 20:7473-7478
Zakrzewicz A, Krasteva G, Wilhelm J et al (2011) Reduced expression of arrestin beta 2 by graft monocytes during acute rejection of rat kidneys. Immunobiology 216:854-861

Zhang H, Trivedi A, Lee JU et al (2011) Matrix metalloproteinase-9 and stromal cell-derived factor- 1 act synergistically to support migration of blood-borne monocytes into the injured spinal cord. J Neurosci 31:15894-15903

Ziegler-Heitbrock L, Ancuta P, Crowe S et al (2010) Nomenclature of monocytes and dendritic cells in blood. Blood 116:e74-e80 